\title{
Investigation of Flexible Clutches Over a Wide Range
}

\author{
Pranav R. Morchapure ${ }^{1}$, Dr K. S. Zakiuddin ${ }^{2}$, Nitin S. Sawarkar ${ }^{3}$ \\ ${ }^{1}$ Research Scholar, Priyadarshini College of Engineering, Nagpur, India, 440019 \\ ${ }^{2}$ Professor, Priyadarshini College of Engineering, Nagpur, India, 440019 \\ ${ }^{3}$ Assistant Professor, Priyadarshini College of Engineering, Nagpur, India, 440019
}

DOI: 10.46335/IJIES.2020.5.10.4

\begin{abstract}
In this study, the use of computer modelling has evaluated an effective and reliable analysis technique for designing versatile clutches. Study based primarily on different forms of Friction Clutches. It is also useful to choose the best suitable clutch for precise equipment.
\end{abstract}

Keywords- Friction Clutch, Analysis.

\section{INTRODUCTION}

$\mathbf{O}$ ne of the most important friction elements for an entire vehicle is the multi-disc clutch, which plays a significant function in transmitting power ${ }^{[1,2]}$. The key role of the clutch is the relation between the driving and driven parts in separate transmissions to be engage and disengaged. To ensure a smooth transfer of power, the clutch is still under slipping condition, which eventually creates a large amount of friction heat. Typically, the slipping of the clutch creates a high surface temperature and a high-temperature gradient, thereby increasing the wear of the clutch and, under extreme conditions, resulting in not only poor shifting efficiency but also eventual clutch failure ${ }^{[3]}$. The friction discs of the clutches undergo significant thermal loading. High temperatures result in failures of thermal stress such as surface cracks, and irreversible distortion. In multidisc clutches, distortion frequently occurs in the form of a conical deformation or disc wave.. Temperatures and stresses were analyze in the friction discs of a wet multidisc clutch ${ }^{[4]}$.

\section{LITERATURE REVIEW}

Abdullah and Schlattmann et al. [1] Research the thermos-elastic behavior of single and multidisc friction clutches during engagement startup. They also examine the effect of pressure on the temperature field between contact surfaces as it varies with time. Two approaches to the internal energy of a clutch disc; heat partition ratio method measures, the heat produced from each component separately, while the second approach uses the touch model to add the cumulative heat generated from the entire component. Also, they studied the effect of time of engagement, the function of sliding speed, thermal load, and Dimensionless disc radius on the thermal action of the friction Clutch during the period of slipping (internal disc radius / outside disc radius). In the sliding process, they concluded that the state of restriction status. The material property is very effective in the distribution of touch pressure and temperature area.

Zou et al. [2], the friction behavior was mentioned briefly during the run-in. Various experiments showed substantial differences in friction behavior during the running-in process. The friction behavior stabilized with a growing duration of the test.

Natsumeda and Miyoshi [3], in terms of computing, Wet-clutch engagement simulation is a relatively mature research topic. The first modelling and hydrodynamic analysis were provide taking into account permeability, rough surface asperity contact, and compressive pressure.

Katsukawa et al. [4] Friction coefficient patterns have seen for friction materials with various types of resins. There were variations, particularly in the running-in process, depending on the resin, both in terms of the development of friction behavior and the level of the friction coefficient. 
Li, T. C., Huang, Y. W., \& Lin, J. F et al. [5] In multidisc clutches, distortion often occurs and is in the shape of a rectangular deformation or disc wave. Temperatures and stresses in the friction discs of a wet multidisc clutch.

Lingesten et al. [6], the purpose of the present study is to suggest a model for the analysis of the wear characteristics of paper-based friction content in a wet clutch subjected to both thermal and mechanical degradation. The analytical technique for predictions of wear proposed to investigate experimentally observed two-stage wear activity.

Deur et al. [7], most wet clutches transfer torque by mechanical friction and fluid interactions. In a compact and lubricant-rich setting, such as automatic transmission, wet clutches are usually use because a wet clutch is generally smaller than a dry clutch. For wet clutches, the maximum transmittable torque per plate pair is limited. Multiple plates are also use to counteract the minimal torque per pair of plates.

J.Y. Jang, M.M. Khonsari et al. [8], a model has developed to investigate the impact on the performance of a wet clutch of radial grooves and waffle-shaped grooves. The convective terms in the oil energy equations are use to determine the temperature fields corresponding to the ranges between grooves. The heat conduction equations in the bounding solids properly formulated.

Li et al. [8], the behavior of crack spread under different braking conditions was investigated, suggesting that high braking energy could accelerate crack spread. In addition to thermal stress, mechanical loads can also cause deformation of the platform.

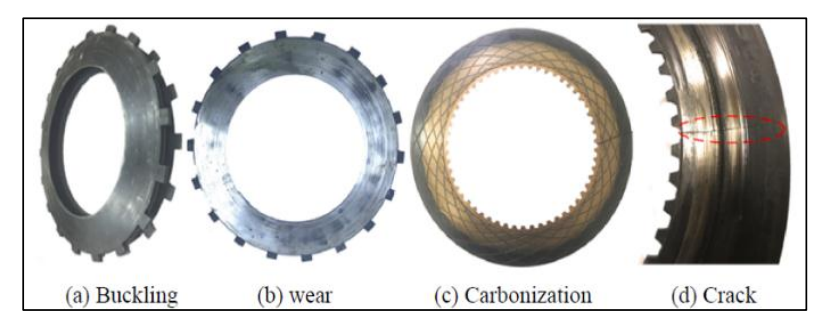

Fig 1: Failures of the friction components ${ }^{[3]}$

\section{A. MULTIPLATE CLUTCH}

To transfer power between the engine shaft and the transmission shaft, the multi-plate clutch uses several clutch plates to make contact with the motor flywheel, as shown in Fig.2. A multi-plate clutch used in cars and machines that needs a high torque output [8]. Multi-plate clutches are use in heavy-duty racecars and motorcycles for high torque transmission. These are smooth and simple to control as opposed to single plate clutches because of their friction surface contact assembly. Nevertheless, little research has carried out to investigate the dynamic characteristics of the clutch when the plate deformation occurs ${ }^{[3]}$.

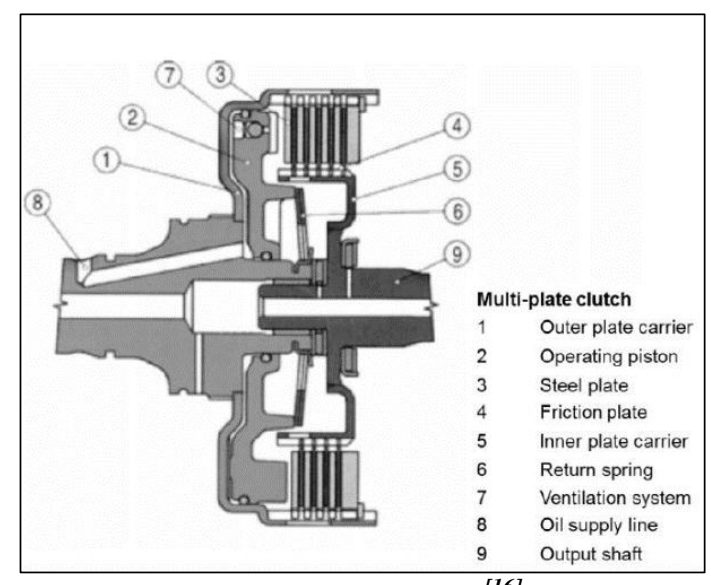

Fig 2: Multiplate Clutch ${ }^{[16]}$

A plate attached to the splined sleeves, which further connected to the pedal fulcrum, as shown in Fig.2. Such as when pressing the clutch pedal, the sleeves connected to the pedal pole shift towards the outside, which drives the pressure plate attached to that splined sleeve in turn. It is a metallic plate having frictional lines at its outer surfaces. Frictional contact with the flywheel is use to transfer power between the engine shaft and the shaft of the transmission.

A vehicle driver-operated clutch pedal used to monitor the engagement and disengagement of the clutch.

Splined Shaft \& Inner Splined Sleeves: An outer splined transmission input shaft over which the complete clutch assembly. That includes clutch plates, pressure plate, inner splined sleeve, the clutch casing place and rotates with it.

It is part of the engine. We can also consider it as part of the clutch system as the transmission of power from the engine output shaft to the transmission shaft obtain by the frictional contact between a clutch and the flywheel of an engine.

\section{B. CENTRIFUGAL CLUTCH}

Where they only have to engage at specified speeds, centrifugal and semi-centrifugal clutches are used. The driving shaft has a spinning member that increases as the shaft speed rises and the clutch engages, which then drives the driven shaft. ${ }^{[8]}$. Clutch friction discs undergo substantial thermal loading. High temperatures, such as surface cracks and irreversible distortions, lead to thermal stress failures ${ }^{[10]}$. 


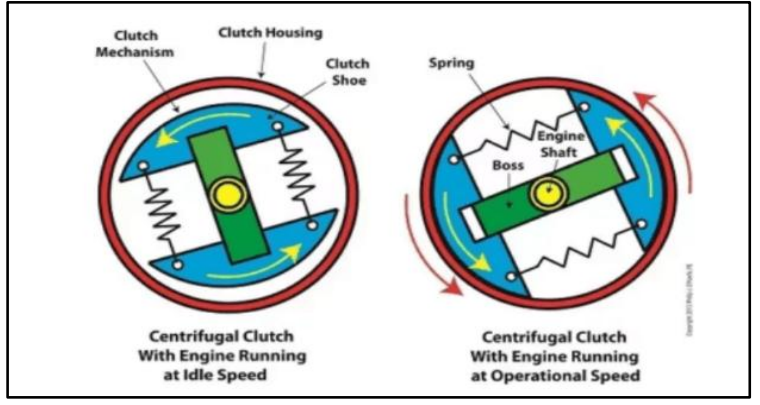

Fig 3: Centrifugal Clutch

Spider or Guide: One of the pressure plates of a simple clutch acts as a spider or guide. It is attached to the engine's driving shaft. Since it keeps the shoe and directs its motion, it is called the guideway. The Springs control the initial location of the shoe in the guide.

Drum: The drum functions as another basic clutch pressure plate. It is connected to and rotates with, the guided member. The drum does not interact specifically with the guide or shoe.

Shoe: Centrifugal clutch is mainly shoe. It functions as an engaging guide and drum member during running conditions. When the driving shaft rotates, the shoe that is free to travel in the guide can be rotated. A centrifugal motion works on it because of rotation which pushes it outward and connects to the drum.

Springs: Springs are controlling element of this type of clutch. Each shoe requires one spring. The shoe is connected with the guide through these springs. It keeps the shoe unlit driving member at its original position at the necessary speed at which the centrifugal force can overpower the spring force ${ }^{[13]}$. If we want to change the engaging speed of the clutch, we simply change these springs with higher spring indexed springs.

The outer face of the shoe that will attach to the drum is fitted with a friction lining. These liners play the same function as the friction plate in a simple clutch. It is used to transmit torque from driving member to driven member.

The centrifugal force at low speed is too low to overcome the spring force, which tends to tie the shoe inside the guide. As the speed increases the shoe move outside and makes a contact with the drum. The friction lining between shoe and drum start to transfer torque from the engine to drum. As the engine speed decreases, the centrifugal force that eliminates drum, shoe contact will decrease, and the clutch will disengage. Therefore, this is a clutch controlled automatically speed. The operating speed of the clutch can maintained by replacing spring.

\section{CONE CLUTCH}

A single type of friction clutch is a cone clutch as shown in Fig.4. Therefore, on the theory of friction, this clutch works. When two rotating surfaces lined with friction material come into contact in rotating state, both of them begin to rotate at the same speed ${ }^{[14]}$. There is an external cone linked to the driving shaft in a cone clutch and an internal cone linked to the driven shaft. There is certain friction lining at the inner part of the outer cone and the outer part of the inner cone. These both cone are bringing in contact to transfer energy from driving shaft to driven shaft.

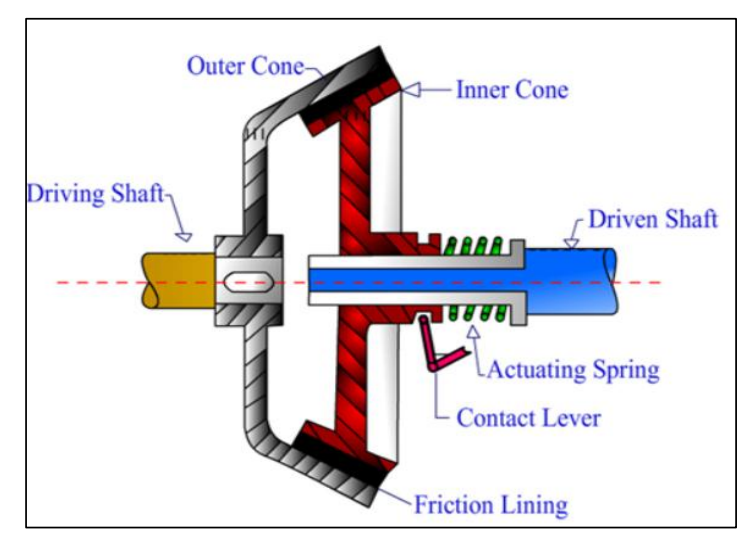

Fig 4: Cone Clutch

Outer Cone: The outer cone is attached to the flywheel or the driven shaft of the engine, often referred to as the female cone. The part of the clutch with the engine shaft is still in rotating condition. The inner portion of the outer cone is line with a friction lining that allows frictional contact with the inner cone and allows the driven shaft to rotate at the same driving shaft speed.

Inner Cone: The inside cone, also called the male cone, is attached to the transmission shaft or gearbox. The friction lining is line with the outer part of the inner cone that makes frictional contact between the inner cone and the outer cone. This part of the clutch remains in contact with the outer cone with the help of pressure springs. If the driver needs to disengage the transmission box from the engine, he moves the pedal of the clutch that disengages the inner cone to the outer cone and disengages Motor inside the Transmission Box.

Pressure Springs: Pressure springs, or simply called springs, are use when the clutch pedal is release to allow contact between male cone and female cone. This spring, which allows the clutch to be disengaged, is compress when the driver presses the clutch pedal.

Sleeves: Part of the clutch on which the inner cone placed during engagement and disengagement of the 
clutch is knows as sleeve and performs to and for motion.

At the starting of the vehicle, the clutch pedal is pressed by the driver which allow compressing the pressure springs thus the inner cone slightly move Near the outer cone. This breaks the link between the inner cone and the outer cone and hence the clutch is in a state of disengagement. Any rotation of the shaft of the engine or driving shaft shall not move to the shaft of transmission or the shaft is driven. Now the driver wants to engage the transmission line thus he slowly released the clutch pedal. This will cause the pressurized spring to expand which moves the inner cone in the direction of the outer cone and cause contact between them. A frictional force acts between the inner side of the outer cone and the outer side of the inner cone. Thus, the engine shaft and transmission shaft allow rotating at the same speed and the clutch is called in engage position.

\section{WET CLUTCH}

A wet clutch transfer's power using mechanical and fluid couplings, by mating rotating friction plates submerged in a lubricant. What differentiates wet clutches from dry clutches is the lubricant between the surfaces ${ }^{[11]}$. In comparison to having no oil in the components, a wet clutch is one that is saturate in oil. It has a set of plates called drive plates that are used in a basket to add drive to the vehicle. A wet clutch consists of a series of discs inside a cage or the clutch for the basket. There are notches on the end of each drive plate that fit into the slots in the clutch basket or cage. The pressure plate assembly, which includes the drive plates and discs, slides into the enclosure to engage it. Since, the clutch basket is connected to the drive chain this drives the vehicle.

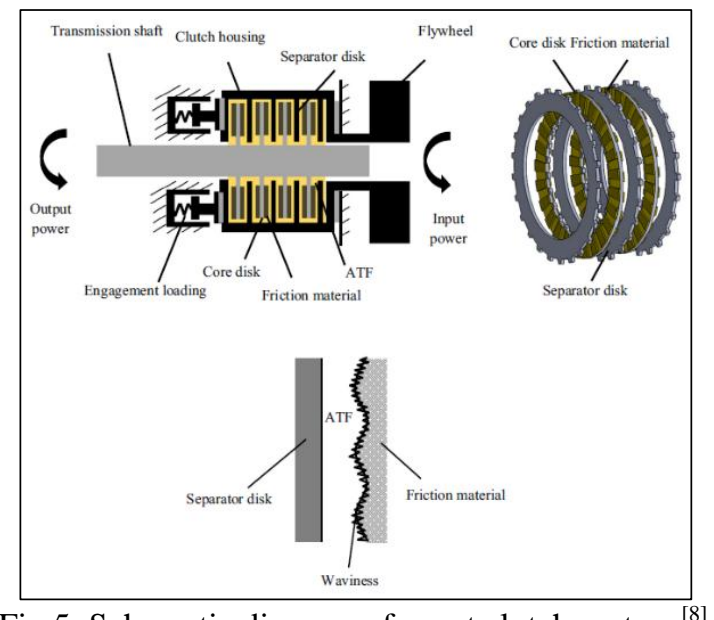

Fig 5: Schematic diagram of a wet clutch system ${ }^{[8]}$

As Shown in fig.5, the amount of friction involved in its operation this entire process is saturated in oil. In a dry clutch the device does not produce any oil. A dry-plate assembly works on friction. A wet clutch uses the friction portion of the oil between the drive plates in order for the plates to slip past each other and for proper operation.

\section{CONCLUSION}

After study, the values of comparable stresses for material loading conditions are understood to be less than the acceptable stresses for the designs under analysis. Thermo-mechanical issues, such as thermal deformation and thermo-elastic instability, can lead to thermal cracking, wear and other clutch disc component failure modes. The friction clutch has some clear demerits like slip, heat between the friction pieces. The issues with slipping are the friction stuff. The friction torque and the temperature growth rate unexpectedly increase, the separate plate certainly experiences elastic deformation. The coefficient of friction that is caused by heat. Which causes the total capacity of the clutch. The findings are very favorable to what was predicted. The stress, deformation and temperature make clear the concept of which parameter should have been taken into account when determining the multi-plate clutch, centrifugal clutch, cone clutch and wet clutch.

\section{REFERENCES}

[1] J. Liu, B. Ma, H. Li, M. Chen, G. Li, Control strategy optimization for a dual-clutch transmission downshift with a single slipping clutch during the torque phase, P. I. Mech. Eng. D - J. Aut. 232 (5) (2018) 651-666.

[2] G. Li, D. Gorges, Optimal control of the gear shifting process for shift smoothness in dual-clutch transmissions, Mech. Syst. Signal. Pr. 103 (2018) 23 38.

[3] Yu, L., Ma, B., Chen, M., Li, H., \& Liu, J. (2020). Investigation on the thermodynamic characteristics of the deformed separate plate in a multi-disc clutch. Engineering Failure Analysis, 110(July 2019), 104385.

[4] Li, T. C., Huang, Y. W., \& Lin, J. F. (2015). Studies on centrifugal clutch of frictional lining materials. Key Engineering Materials, 642, 39-44.

[5] Abdullah O I, Akhtar M J, Schlattmann J. Investigation of thermo-elastic behavior of multidisc clutches. $J$ Tribol 137(1): 1-9(2015)

[6] E Zhao, B Ma, H Li. The tribological characteristics of Cu-based friction pairs in a wet multidisc clutch under nonuniform contact. ASME Journal of Tribology, 2018, 140(1)

[7] S. Natsumeda, T. Miyoshi, Numerical simulation of engagement of paper based wet clutch facing, J. Tribol.116(1994)232-237. 
[8] Z. Li, J. Han, Z. Yang, L. Pan, The effect of braking energy on the fatigue crack propagation in railway brake discs, Eng. Fail. Anal. 44 (2014) 272 -284.

[9] Li, M., Khonsari, M. M., McCarthy, D. M. C., \& Lundin, J. (2015). On the wear prediction of the paperbased friction materialin a wet clutch. Wear, 334335(April), 56-66.

[10] M Katsukawa. Effects of the physical properties of resins on friction performance. SAE Technical Paper No. 2019-01-0341, 2019.

[11] T.P. Newcomb and R.T. Spurr: Friction materials (Tribology Handbook, Butterworths, London 1973).

[12] J. Choi, M. Nakhaeinejad, M. Bryant, Simulation of wet clutch engagement on electronically-controlled limited-slip differential with consideration of degraded clutch performance. "in Proceeding. of International Joint Tribology Conference ID-41265, (San Francisco, CA, 2010)

[13] J. Deur et al., Modeling of Wet Clutch Engagement Including a Thorough Experimental Validation, SAE Int. 2005-01-0877, 2005.

[14] J.Y. Jang, M.M. Khonsari, Three-dimensional thermohydrodynamic analysis of a wet clutch with consideration of grooved friction surfaces. ASME J. Tribol. 133, 011703-1-011703-12 (2011)

[15] M T Devlin, SH Tersigni, J Senn, et al. Effect of friction material on the relative contribution of thinfilm friction to overall friction in clutches. SAE Technical Paper No. 2004-01-3025, 2004.

[16] M. Li, M.M. Khonsari, D.M.C. McCarthy, J. Lundin, Parametric analysis of wear factors of a wet clutch friction material with different groove patterns, P. I. Mech. Eng. J - J. Eng. 231 (8) (2017) 1056-1067.

[17] Hirz, M. (2018). Dynamic simulation of multi-plate clutches for automotive applications Authors. March. 